\title{
Síntomas somáticos en cuidadores de pacientes geriátricos con o sin sobrecarga, del área urbano-marginal Payet, Independencia, Lima, Perú
}

\author{
Somatic symptoms in overloaded or not overloaded caregivers of geriatric patients in an urban-rural area \\ of Payet, Independencia, Lima
}

Zoila Romero ${ }^{1}$, María Cuba ${ }^{2}$

\section{RESUMEN}

Aunque es asumido que la presencia de sobrecarga del cuidador de pacientes geriátricos predispone al desarrollo de síntomas somáticos, dicho problema no ha sido estudiado en el Perú y más aún en un área urbano-marginal, por lo que se necesitan más estudios que validen esta asociación. Objetivo: Determinar la relación entre el desarrollo de síntomas somáticos y la presencia de sobrecarga en cuidadores de pacientes geriátricos del Área urbano-marginal Payet, Independencia. Material y métodos: Estudio de relación, transversal y basado en la aplicación de escalas de medición, aplicadas a 93 cuidadores de pacientes geriátricos. Se analizó con el programa SPSS versión 18.0 y aplicando el test de proporciones. Resultados: El 77,4\% tenía entre 25 a 59 años. El 61,29\% de los cuidadores tenía un tiempo entre 1 a 8 años cuidando pacientes geriátricos y el 67,74\% empleaba menos de 8 horas diarias en el cuidado. El 90,3\% de los cuidadores no había recibido capacitación para el cuidado. El 75,27\% de los cuidadores presentaron sobrecarga intensa y el 40,86\% presentó alta severidad de los síntomas somáticos, encontrándose asociación estadística entre estas dos variables. Conclusiones: Se encontró relación entre el desarrollo de síntomas somáticos y la presencia de sobrecarga en los cuidadores de pacientes geriátricos del área urbano-marginal de Payet.

PALABRAS CLAVE: Cuidadores, adulto mayor, trastornos somatoformes. (Fuente: DeCS BIREME)

\section{SUMMARY}

It is assumed that work overload in caregivers of geriatric patients predisposes them to develop somatic symptoms. However, this relationship has not been well studied in. Objective: Determine the relationship between development of somatic symptoms and work overload in caregivers of geriatric patients in the district of Independencia. Methods: Cross-sectional study based on the application of measurement scales to 93 caretakers of geriatric patients. Comparison of proportions was made using the statistical package SPSS version 18.0. Results: $77,4 \%$ of participants had 25-59 years of age; $61,29 \%$ worked as caregivers for 1-8 years, and 67,74\% of them worked less than 8 hours daily. No previous training had been received in $90,3 \%$ of caregivers. Intense work overload was observed in $75,27 \%$, and $40,86 \%$ had severe somatic symptoms. These two variables were statistically associated. Conclusions: An association between work overload and development of somatic symptoms among caregivers of

1 Médica Familiar. Equipo técnico Sub Gerencia de Promoción de la Salud. Gerencia de Prestaciones Primarias de Salud. EsSalud, Lima, Perú.

2 Médica Familiar. Jefe Médico del Policlínico Juan José Rodríguez Lazo, Coordinadora de la especialidad de Medicina Familiar de la Universidad Peruana Cayetano Heredia y Presidenta de la Sociedad Peruana de Medicina Familiar y Comunitaria, Lima, Perú. 
geriatric patients in the urban-rural area of Payet was found.

KEYWORDS: Caregivers, elderly, somatoform disorders. (Source: MeSH NLM)

\section{INTRODUCCIÓN}

El paciente geriátrico tiene generalmente alguna alteración que limita su capacidad tanto para realizar sus actividades básicas como instrumentales de la vida diaria, llevándolo por diferentes motivos a depender de otras personas, un cuidador, el que se convierte, en nexo primordial entre los profesionales de salud y el paciente. El cuidador es un elemento fundamental en la prevención, diagnóstico precoz, tratamiento y rehabilitación del adulto mayor (1). En 1989 Lawton y col (2), lo identificaron como la persona que más tiempo se dedica a proveer los cuidados que precisa su familiar. En estudios en países desarrollados, los cuidadores familiares proveen casi el $80 \%$ del cuidado que reciben los adultos mayores en la comunidad (1).

El ser cuidador es un riesgo de problemas en su propia salud (1), pueden experimentar problemas graves de salud física y mental derivados del estrés por las circunstancias en que se desarrolla el cuidado. En si la carga del cuidador engloba múltiples dimensiones por lo que, este término no puede ser resumido en un único concepto sino que tiene que ser entendido dentro de un proceso multidimensional $(3,4)$.

Muchas veces los síntomas que desarrollan los cuidadores sometidos a sobrecarga no son detectados en la consulta de Atención Primaria y por lo general carecen de explicación clínica definida; muchos pacientes presentan estos síntomas de modo incidental pero otros los padecen crónicamente y pasan mucho tiempo buscando atención médica (5).

Los síntomas somáticos en estos pacientes son expresiones de dificultades emocionales que resumen complejas interacciones psicológicas, vitales, familiares y sociales, difíciles y estresantes para el sujeto. Este heterogéneo cuadro recibe distintos nombres: somatizaciones, cuadros funcionales $\mathrm{o}$ síntomas físicos sin explicación médica. Aplicado a estos sujetos, el modelo biomédico obtiene resultados insatisfactorios. La alta utilización de recursos, estudios innecesarios e interconsultas a especialistas genera altos costos $\mathrm{y}$ riesgo de complicaciones iatrogénicas (6).

Se estima que la prevalencia de síntomas somáticos no explicados como causa de consulta externa en atención primaria es entre 15 y $25 \%$ y de estos un 30 a $70 \%$ no tienen explicación luego de una evaluación (5).

No existen estudios en nuestro medio que evalúen la relación entre el desarrollo de síntomas somáticos y la presencia de sobrecarga en cuidadores de pacientes geriátricos; y dado que en nuestro país ha habido crecimiento de la población adulta mayor, el sistema de atención en salud no ofrece las condiciones para que la población que se encarga del cuidado de estos pacientes reciba la atención o la capacitación que requieren, más en los lugares más apartados de nuestro país y en zonas de mayor índice de pobreza.

El objetivo del estudio fue determinar la relación entre el desarrollo de síntomas somáticos y la presencia de sobrecarga en cuidadores de pacientes geriátricos del Área Urbano Marginal Payet - Independencia.

\section{MATERIAL Y MÉTODOS}

Estudio de relación y de corte transversal, realizado en el entorno del Puesto de Salud José Olaya, ubicado en Payet, distrito de Independencia; este tiene dentro de su jurisdicción 15 asentamientos humanos, siendo su población total de 9858 , de los cuales 1088 corresponden a adultos mayores.

La población estuvo conformada por individuos mayores de 18 años, de ambos sexos, cuidadores de adultos mayores de la comunidad.

Se calculó el tamaño de la muestra mínimo en 93 pacientes, considerando un $\alpha$ de 0,05 y un $\beta$ de 0,20 . El muestreo fue no probabilístico por conveniencia, realizado entre julio y septiembre del 2012, a través de visita domiciliaria, el cual fue efectuado por la investigadora.

La evaluación de los participantes se realizó previo consentimiento informado. Los datos se registraron en una ficha de recolección e incluía edad, sexo, estado civil, grado de instrucción, tipo de seguro de salud, parentesco, capacitación, tiempo y horas dedicadas al cuidado del adulto mayor: en estas dos últimas, se tuvo en cuenta las clasificaciones utilizadas en trabajos de investigación realizados en otros países, no existiendo en la literatura puntos de cortes para ello. 
El estudio fue aprobado por el comité de ética de la Universidad Peruana Cayetano Heredia.

Sobrecarga del cuidador se definió como la medida de carga a la que está expuesto el cuidador del paciente adulto mayor; se utilizó la Escala de Zarit, validada para nuestro medio (7). La escala consta de 22 preguntas, que evalúan calidad de vida, capacidad de autocuidado, red de apoyo social y competencias para afrontar problemas conductuales y clínicos del paciente al cuidado; tiene una sensibilidad de $84,5 \%$ y una especificidad de $85,3 \%$ para ausencia de sobrecarga y una sensibilidad de $89,7 \%$ y una especificidad de $94,2 \%$ para sobrecarga intensa; esta escala clasifica a los participantes con ausencia de sobrecarga (22 46 puntos), sobrecarga ligera ( $47-55$ puntos) y con sobrecarga intensa $(56-110$ puntos $) .(4,7)$.

Se definió síntomas somáticos, a los síntomas sin explicación clínica definida; para su evaluación se empleó la Escala PHQ - 15 (Patient Health Questionnaire) (8), que evalúa la severidad de los síntomas somáticos. Esta escala consta de 15 preguntas que evalúan aproximadamente el $90 \%$ de las quejas físicas del paciente (9); tiene una sensibilidad de $78 \%$ y una especificidad de $71 \%(9,10)$. La escala ha sido validada al idioma castellano en España por Ros y col (11). Para definir mínima gravedad, baja gravedad, gravedad media y alta severidad de los síntomas somáticos, se tuvo en cuenta la puntuación obtenida en la escala, esto es 0-4 puntos, 5-9 puntos, 10-14 puntos y 15 o más puntos respectivamente.

\section{Análisis estadístico}

Se realizó estadística descriptiva, los datos fueron expresados en frecuencias. Se utilizó la prueba de chi cuadrado, para determinar la asociación entre sobrecarga del cuidador y el desarrollo de síntomas somáticos en pacientes cuidadores de adultos mayores. Se consideró significativo un $\mathrm{p}<0,05$. Se utilizó el Programa SPSS v. 18.0 para Windows, previa codificación de los datos.

\section{RESULTADOS}

Las características de la población estudiada se muestran en la Tabla 1.

Ochenta y cuatro $(90,3 \%)$ cuidadores presentaban algún grado de sobrecarga $(75,2 \%$, sobrecarga intensa y $15,1 \%$, sobrecarga ligera); todos tenían síntomas somáticos, $38(40,9 \%)$ presentan alta severidad, 35 $(37,6 \%)$ gravedad media y $20(21,5 \%)$ baja severidad
Tabla 1. Características demográficas y socioeconómicas de la población de estudio.

\begin{tabular}{lcr}
\hline & n & $\%$ \\
\hline Sexo & 34 & 36,6 \\
Masculino & 59 & 63,4 \\
Femenino & & \\
Edad & 7 & 7,5 \\
18 - 24 años & 72 & 77,4 \\
$25-59$ años & 14 & 15,1 \\
$60-80$ años & 14 & 0 \\
80 años a más & 0 &
\end{tabular}

Estado civil

Soltero

Casado

Divorciado o viudo

Conviviente

Grado de instrucción

Iletrado

Primaria incompleta

Primaria completa

Secundaria incompleta

Secundaria completa

Superior incompleta

Superior completa

Seguro social

SIS

EsSalud

Fuerzas Armadas

Otros

Tipo de cuidador

Familiar $(*)$

Esposo

Hermano

Hijo

Nieto

Sobrino

No familiar

Contratado o voluntario

Tiempo al cuidado del paciente geriátrico

Menos de 1 año

1 a 8 años

Más de 8 años

No especifica tiempo

Horas diarias al cuidado del paciente geriátrico

Menos de 8 horas/día

Más de 8 horas/día

No especifica cantidad de horas

$17 \quad 18,3$

$74 \quad 79,6$

0

2,2

6,5

16,1

40,9

1,1

28,0

3,2

4,3

64,5

33,3

1,1

1,1

97,9

23,7

6,4

47,3

10,8

7,6

2,1

0

Capacitación para el cuidado del paciente geriátrico

\begin{tabular}{lrr}
$\mathrm{Si}$ & 9 & 9,7 \\
$\mathrm{No}$ & 84 & 90,3 \\
\hline
\end{tabular}

$\left({ }^{*}\right)$ En esta categoría se ha considerado como familiar: esposo, hermano, hijo, nieto y sobrino. 
Tabla 2. Relación entre sobrecarga del cuidador y desarrollo de síntomas somáticos en pacientes cuidadores de pacientes geriátricos.

\begin{tabular}{|c|c|c|c|c|c|c|c|c|}
\hline \multirow{3}{*}{ Sobrecarga } & \multicolumn{6}{|c|}{ Severidad de síntomas somáticos } & & \\
\hline & \multicolumn{2}{|c|}{ Baja severidad } & \multicolumn{2}{|c|}{ Gravedad media } & \multicolumn{2}{|c|}{ Alta severidad } & \multicolumn{2}{|c|}{ TOTAL } \\
\hline & $\mathbf{n}$ & $\%$ & $\mathbf{n}$ & $\%$ & $\mathbf{n}$ & $\%$ & & \\
\hline Ausencia de sobrecarga & 2 & 10,0 & 6 & 17,2 & 1 & 2,6 & 9 & 9,7 \\
\hline Sobrecarga ligera & 3 & 15,0 & 9 & 25,7 & 2 & 5,3 & 14 & 15,1 \\
\hline Sobrecarga intensa & 15 & 75,0 & 20 & 57,1 & 35 & 92,1 & 70 & 75,2 \\
\hline Total & 20 & 21,5 & 35 & 37,6 & 38 & 40,9 & 93 & \\
\hline No hubo casos de baja se & & & & & & & & \\
\hline
\end{tabular}

$\mathrm{p}=0,015155$

de los síntomas somáticos.

Se encontró relación entre el tiempo al cuidado del adulto mayor y la presencia de sobrecarga del cuidador $(\mathrm{p}=0,0252)$, no así con el desarrollo de síntomas somáticos. No se encontró relación entre el número de horas dedicadas al cuidado del adulto mayor y la presencia de sobrecarga en los cuidadores, tampoco con el desarrollo de síntomas somáticos.

Se encontró asociación estadísticamente significativa entre la presencia de sobrecarga y el desarrollo de síntomas somáticos, $(\mathrm{p}=0,015155)$, (Tabla 2).

\section{DISCUSIÓN}

El estudio, buscó determinar la asociación entre la presencia de sobrecarga del cuidador y el desarrollo de síntomas somáticos, encontrándose relación significativa entre ambos.

Nuestro estudio coincide con los realizados en México (12), en Cuba, (13), en España (14), y en Perú $(15,16)$ en relación a que el cuidador principalmente es del sexo femenino y que generalmente es una persona adulta la que asume esta responsabilidad. En relación al tipo de cuidador, este estudio coincide con estudios realizados en México (12) y Cuba (13), en el que se evidenciaba que generalmente eran familiares, a predominio del hijo, quienes asumían el cuidado del adulto mayor, pero difiere de un estudio realizado en nuestro medio (15), que encontró que el tipo de cuidador era generalmente la cónyuge, esto debido quizás a la diferencia de pobladores entre los distritos de Lima.

En cuanto al grado de instrucción, coincidimos con estudios realizados en España y en nuestro medio, en donde se evidencia que en su mayoría eran cuidadores que sólo poseían nivel primario de instrucción $(14,15)$.
En cuanto al tiempo al cuidado del adulto mayor, un estudio evidenció 3 años (14) y en un nuestro medio, entre 5 a 9 años (15); estos datos coinciden con lo encontrado en nuestro estudio, en el que se encontró entre 1 a 8 años en la mayoría de los casos. El número de horas diarias dedicadas al cuidado del adulto mayor, encontrado en nuestro estudio difiere de otro estudio realizado en nuestro medio, en el que se encontró el uso de 160 horas/semana (15); esto puede deberse, a que la población encuestada era de bajos recursos económicos y que dedican la mayor parte del tiempo a trabajar fuera del hogar, relegando el cuidado del adulto mayor a periodos de tiempo de ocio.

Se considera importante el hecho de que la mayor parte de cuidadores no posean capacitación en el cuidado del adulto mayor, ya que el poco dominio en el cuidado de esa población, así como del manejo del estrés, traen como consecuencia una atención de mala calidad hacia el adulto mayor y que surjan problemas tanto físicos, como mentales en los cuidadores; no existen estudios al respecto, pero es conveniente se pudieran desarrollar a futuro.

Al evaluar la presencia de sobrecarga del cuidador, los resultados de nuestro estudio son similares con los de dos estudios en México, que encontraron 73,92\% y $66,4 \%$ de sobrecarga en los cuidadores $(17,18)$. Otros estudios realizados en España (19), en México, (20), en Colombia, (21) y en nuestro medio (16), han encontrado entre 30 y $50 \%$ de cuidadores con sobrecarga; esto puede deberse, al grado de dependencia y la presencia de patologías concomitantes que presentaban los adultos mayores, así como otros factores que tienen que ver con el bajo nivel socioeconómico que poseen los cuidadores; se recomendaría mayores estudios que relacionen estas variables.

Con respecto a la presencia de síntomas somáticos, nuestro estudio difiere de los resultados encontrados en otros países como Australia, en donde se encontró 
que el $18,5 \%$ de los pacientes eran somatizadores (22) y en Alemania, donde la prevalencia de desórdenes somatomorfos era de $22,9 \%$ (23); en nuestro medio no se encuentran estudios que valores la presencia de síntomas somáticos, esto es importante ya que nos lleva a plantear que una alta frecuencia de casos implica mayor investigación al respecto.

Se encontró relación entre la presencia de sobrecarga y el desarrollo de síntomas somáticos, en la población estudiada; al respecto no se encontraron estudios a nivel internacional, ni nacional que relacionen estas dos variables, pero de acuerdo a lo establecido por Pérez y col (1996), el cuidador con sobrecarga se caracteriza por la existencia de un cuadro plurisintomático que suele afectar y repercutir en todas las esferas de su persona (24) y a lo referido por Ribas y col en España, que los cuidadores presentan una frecuencia del $70 \%$ de patologías psiquiátricas (25), se recomienda mayor investigación al respecto, considerándose de importancia, ya que las patologías mentales deterioran no solo la calidad de vida de los cuidadores, sino que esto repercute en el cuidado del adulto mayor a su cargo.

En relación al tiempo al cuidado del adulto mayor y el número de horas diarias dedicadas al cuidado del adulto mayor y su relación con la presencia de sobrecarga y síntomas somáticos; un estudio en México encontró un mayor nivel de sobrecarga en cuidadores que tenían menos antigüedad laboral (26), lo que lleva a plantearnos que se requiere mayor investigación al respecto.

Se debe plantear para futuras investigaciones evaluar el problema considerando una población mayor y realizando la comparación en diferentes escenarios, esto es, con diferencias sociodemográficas y culturales; además de considerar la presencia de comorbilidades en los adultos mayores; que nos ayudaría a determinar qué grado de influencia ejercen en los resultados. Otro aspecto a considerar es que, si bien el PHQ - 15 es una escala que mide la severidad de los síntomas somáticos, para realizar el diagnóstico de patología somática se deben tener en cuenta los criterios de la DSM IV o CIE 10, lo que puede ser tomado en cuenta para futuras investigaciones.

Hubo limitaciones en el estudio, entre las que destacamos la poca accesibilidad geográfica, cultural e idiosincrática tanto de los pacientes geriátricos, como de los cuidadores y la inexistencia de patrones que documenten fehacientemente la cantidad de adultos mayores dentro de la comunidad.

En suma, todos estos aspectos, nos llevan a plantearnos que como personal de salud que trabaja en Atención Primaria debemos incorporar dentro de nuestros programas de atención al adulto mayor estrategias que incluyan a los cuidadores, a fin de brindar una mayor calidad de vida a ambos.

En conclusión existe asociación entre la presencia de sobrecarga y el desarrollo de síntomas somáticos, así como con el tiempo al cuidado el adulto mayor.

\section{Declaración de financiamiento y de conflictos de intereses:}

Los autores declaran no tener conflictos de intereses. El estudio fue financiado por los investigadores.

\section{Correspondencia:}

Zoila Olga de los Milagros Romero Albino

Calle San Borja Norte 1308, Dpto. 301 - San Borja, Lima, Perú.

Correo electrónico: zoilara80@hotmail.com

Celular: 981604088 - 974629788

\section{REFERENCIAS BIBLIOGRÁFICAS}

1. Parodi JF. El cuidador del adulto mayor dependiente. En: Varela L. Principios de Geriatría y Gerontología. 1era edición. Lima: Centro Editorial de la Universidad Peruana Cayetano Heredia; 2003. p. 34-38.

2. Lawton MP, Kleban MH, Moss M, Glicksman A. Measuring caregiving appraisal. J Gerontol. 1989; 46:61-71.

3. Zarit SH. Caregiver's Burden. In: Andrieu S, Aquino JP. Family and professional carers: findings lead to action. Paris: Serdi Edition and Fondation Médéric Alzheimer; 2002. p. 167-190.

4. Carretero S, Garcés J, Rodenas F. La sobrecarga de los cuidadores de personas dependientes: análisis y propuestas de intervención psicosocial (Internet). Valencia; 2005. (Citado el 11 de marzo del 2013) Disponible en: http://www.imsersomayores.csic.es/ documentos/documentos/polibienestarsobrecarga-02.pdf

5. Rubinstein A, Terrasa S. Pacientes con síntomas somáticos no explicables. En: Rubinstein A. Medicina Familiar y Práctica Ambulatoria. Buenos Aires: Editorial Panamericana. 2006: p. 273-279.

6. Chávez H, Tello T. El cuidador del adulto mayor. En: Varela L. Principios de Geriatría y Gerontología. 1era edición. Lima: Centro Editorial de la Universidad 
Peruana Cayetano Heredia; 2003. p. 389-394.

7. Breinbauer H, Vásquez H, Mayanz S, Guerra C, Millán T. Validación en Chile de la Escala de Sobrecarga del Cuidador de Zarit en sus versiones original y abreviada. Rev Méd Chile. 2009; 137: 657665.

8. Interian A, Allen L, Gara M, Escobar J, DíazMartínez A. Somatic complaints in primary Care: Further Examining the validity of the patient health questionnaire (PHQ-15). Psychosomatics. 2006; 47:392-398.

9. Van Ravesteijn H, Wittkampf K, Lucassen P, et al. Detecting somatoform disorders in primary care with the PHQ-15. Ann Fam Med. 2009; 7:232-238.

10. Kroenke K, Spitzer R, Williams J. The PHQ-15: Validity of a New Measure for Evaluating the Severity of Somatic Symptoms. Psychosomatic Medicine. 2002; 64:258-266.

11. Ros S, Comas A, Garcia-Garcia M. Validación de la versión española del cuestionario PHQ-15 para la evaluación de síntomas físicos en pacientes con trastornos de depresión y/o ansiedad: Estudio DEPRE-SOMA. Actas Españolas de Psiquiatría. 2010; 38(6):345-357.

12. Morales E, Enriquez M, Jimenez B, Miranda A, Serrano T. Evaluación del colapso del cuidador en el paciente adulto mayor con osteoartrosis. Rev Mex Med Fis Rehab. 2010; 22(3):96-100.

13. Lara L, Díaz M, Herrera E, Silveira P. Síndrome del cuidador en una población atendida por equipo multidisplinario de atención geriátrica. Rev Cub Enferm. 2001; 17(2): 107-11.

14. Regueiro A, Pérez A, Gomara S, Ferreiro C. Escala de Zarit reducida para la sobrecarga del cuidador en atención primaria. Aten Primaria. 2007; 39(4)185-8.

15. Parodi JF. Perfil del cuidador del paciente adulto mayor dependiente del Centro Geriátrico Naval. Tesis para optar el Titulo de Geriatra. Lima, Perú. Universidad Nacional Mayor de San Marcos, 2000. pp 43.

16. Jiménez M. Perfil epidemiológico del cuidador en el Servicio de Atención Domiciliaria Geriátrica de la clínica geriátrica "San José” PNP. Tesis para optar el Titulo de Geriatra. Lima, Perú. Universidad Nacional Mayor de San Marcos, 2003. pp 36.

17. Navarro A. Relación entre personalidad, sobrecarga y estrategias de afrontamiento en cuidadoras de pacientes de Pacientes mayores dependientes en el área hospitalaria Virgen Macarena de Sevilla. Nure Investigación. 2011; 51:1-11.

18. López J, Orueta R, Gómez-Caro S, Sánchez A, Carmona J, Alonso F. El rol del cuidador de personas dependientes y sus repercusiones sobre su calidad de vida y su salud. Rev Clin Med Fam. 2009; 2(7):332334.

19. Pérez A. Dependencia, cuidado informal y función familiar. Análisis a través del modelo sociocultural de estrés y afrontamiento. Tesis doctoral. Salamanca, España. Universidad de Salamanca, 2008. pp 214.

20. Camacho L, Hinostrosa G, Jiménez A. Sobrecarga del cuidador primario de personas con Deterioro Cognitivo y su relación con el Tiempo de Cuidado. Revista Enfermería Universitaria ENEO-UNAM. 2010; 7(4):35-41.

21. Dueñas E, Martínez A, Morales B, Muñoz C, Viáfara A, Herrera J. Síndrome del cuidador de adultos mayores discapacitados y sus implicaciones psicosociales. Colomb Med. 2006; 37(2):31-38.

22. Clarke D, Piterman L, Byrne C, Austin D. Somatic symptoms, hypochondriasis and psychological distress: a study of somatization in Australian general practice. MJA. 2008; 189: 560-564.

23. Steinbrecher N, Koerber S, Frieser D, Hiller W. The prevalence of medically unexplained symptoms in primary care. Psychosomatics. 2011; 52(3):26371.

24. Pérez JM, Abanto J, Labarta J. El síndrome del cuidador en los procesos con deterioro cognoscitivo (demencia). Atención Primaria. 1996; 18(4):82-98.

25. Ribas J, Castel A, Escalada B, et al. Trastornos psicopatológicos del cuidador principal no profesional de pacientes Ancianos. Rev Psiquiatría Fac Med Barna. 2000; 27(3):131-134.

26. Hernández Z, Ehrenzweig Y. Percepción de sobrecarga y nivel de burnout en cuidadores formales del adulto mayor institucionalizado. Enseñanza e Investigación en Psicología. 2008; 13: 127-142.

Recibido: 11/03/2013

Aceptado: 23/08/2013 